\title{
Distribution and Virulence Phenotypes of Soybean Cyst Nematode (Heterodera glycines) based upon Host Differentials in Jilin Province
}

\author{
Xiujuan Yan ${ }^{1,2 \dagger}$, Jinwen Liu ${ }^{2 \dagger}$, Mingshu $\mathrm{Li}^{2}$, Qiang Qiu ${ }^{2}$, Xiaofeng Zhu ${ }^{1}$ and Yuxi Duan ${ }^{1 *}$ \\ ${ }^{1}$ Nematology Institute of Northern China, Shenyang Agricultural University, Shenyang 110161, China \\ ${ }^{2}$ Soybean Research Institute, Jilin Academy of Agricultural Sciences Changchun 130124, China \\ *For correspondence: duanyx6407@163.com \\ † Contributed equally to this work and are co-first authors \\ Received 02 December 2020; Accepted 18 December 2020; Published 25 January 2021
}

\begin{abstract}
Jilin is the dominant soybean production province in China. Soybean cyst nematode [Heterodera glycines] (SCN) is one of the most important yield-limiting factors in soybean production. Information about the distribution and virulence phenotypes of SCN in soybean fields are essential for optimizing varieties choice in the region. The distribution and virulence phenotypes of SCN in 141 soil samples from 38 cites (cities, counties and towns) across Jilin province were investigated. One hundred and four $(73.76 \%)$ of the samples from all 38 cities (counties, towns) tested positive for SCN and SCN population densities were more than 5 cysts $/ 100 \mathrm{~mL}$ soil in 53 samples from 27 cities (counties, towns). In those 53 samples, we identified 7 races and 12 Heterodera glycines (HG) types, with Race 3 and HG Type 7 being the most dominant genotypes. Of all the genotypes identified, Race10 and HG Type 1.7 were found for the first time in China, and HG Type 3.4.5.7 was the first reported worldwide. Thirty-eight SCN populations (34.5\%) were virulent on plant introduction (PI) 548316 (\#7) and 15 of them had female indices $(\mathrm{FI}) \geq 10 \%$ on Pickett. PI 548316 and Pickett were not recommended as parents of breeding against cyst nematode in Jilin province. Peking-type resistance sources were preferred to pi88788-type in Jilin province. In addition, it was found neither the race nor HG scheme is sufficient for differentiating SCN populations in Jilin province, nor the combination of the two methods is recommended for studying the genetic diversity of SCN in Jilin province. That is, Pickett which was removed in HG scheme should be included not as an indicator line but just to separate different races from the same HG type. (C) 2021 Friends Science Publishers
\end{abstract}

Keywords: Parasite; Virulence phenotypes; Cyst isolation

\section{Introduction}

Soybean cyst nematode ( $\mathrm{SCN}$ ) is an obligate parasite, and its host specialization is obvious. The diversity of $\mathrm{SCN}$ is evaluated either by the race determination scheme (Golden et al. 1970; Riggs and Schmitt 1988) or HG Type classification scheme (Niblack et al. 2002).

The Race determination scheme was developed in 1970 (Golden et al. 1970); where four differential soybean lines including Pickett, Peking, PI 88788 and PI 90763 and the susceptible cultivar Lee 68 was used to characterize the heterogeneity of $H$. glycines. Based on this scheme, 16 potential races were proposed (Riggs and Schmitt 1988). Currently, it had become international scheme for classifying $H$. glycines virulence. Of the 16 races, race 11 and race 13 have not been found yet, and race 16 was reported only once. There are 13 races in the United States, including race $1,2,3,4,5,6,7,8,9,10,12,14$ and 15 (Kim et al. 1997; Xu et al. 2010). Nine races 1, 2, 3, 4, 5 , 6, 7, 9 and 14 had been found in China with 1, 3 and 4 being the most widely distributed (Song et al. 2016). In 2017, a new SCN population (called race X12) was detected in Shanxi province, China (Lian et al. 2017). With more and more new SCN populations identified and reported, the race scheme became not sufficient to classify the virulence profile in SCN. Therefore, a new scheme for the virulence phenotype-HG Type classification was proposed (Niblack et al. 2002). HG Type testing enables more accurate management recommendations than the Race scheme does (Winter et al. 2006). Since the scheme was formally proposed at the national soybean cyst nematode conference in 2008, it has been widely accepted and used. Virulence phenotypes of $H$. glycines had been surveyed with HG Type in some U.S. states (Niblack et al. 2003; Zheng et al. 2006; Mitchum et al. 2007; Colgrove and Niblack 2008; Rzodkiewicz 2010; Acharya et al. 2016; Howland et al. 2018) and also in Korea (Kim et al. 2013). In China, the race scheme has always been used, and the application of HG scheme is relatively less (Wang et al. 2014; Chen et al. 2015; Cui et al. 2018).

To cite this paper: Yan X, J Liu, M Li, Q Qiu, X Zhu, Y Duan (2021). Distribution and virulence phenotypes of soybean cyst nematode (Heterodera glycines) based upon host differentials in Jilin province. Intl J Agric Biol 25:735-741 
In China, most soybeans are produced in Jilin province and the SCN disease spreads widely in this area. The lack of distribution and phenotypic diversity surveys hinder the efficient management of SCN. The only study on the distribution of SCN in Jilin province was reported in 1988 (Liu and Wu 1988); In the past 30 years, there was no report about SCN distribution and genetic diversity of SCN population in Jilin Province, which seriously restricted the progress of soybean resistance breeding against cyst nematode. There are only a few resistant varieties bred from limited resistant sources available on the market, e.g., Bainong 8, Bainong 9 and Bainong 10 derived from Peking. Once SCN disease breaks out, only a few resistant varieties would not be enough.

The objective of this study is to determine the distribution, density, as well as the diversity of virulence phenotypes of $H$. glycines in Jilin province, thus to provide theoretical foundation for introducing and breeding suitable resistant varieties in the region.

\section{Materials and Methods}

\section{Soil sample collection}

Soil samples were collected from soybean fields in Jilin province. A total of 141 fields from 38 cities (counties, towns) were sampled after harvest (Table 1) via multi-point random sampling within the circumference of the soybean roots. The soil samples were divided into two parts, one for counting to determine the density of the cysts, the other were planted with the $\mathrm{SCN}$-susceptible soybean Jiyu 86 to increase population densities for studying virulence phenotypes of SCN.

\section{SCN populations}

Soil samples with population of more than 5 cysts $/ 100 \mathrm{~mL}$ soil were further tested for distribution of virulence phenotypes by race scheme and HG Type scheme.

\section{Soybean differential lines}

The four indicator lines Pickett, PI 548402(Peking), PI 88788 and PI 90763 and susceptible cultivar Lee 74 were from Soybean Research Institute, Jilin Academy of Agricultural Sciences. The other indicator lines PI 548402, PI 437654, PI 209332, PI 89772 and PI 548316, were from Nematology Institute of Northern China, Shenyang Agricultural University.

\section{Cyst isolation}

The isolation of cyst was carried out as described previously (Liu 1995). In brief, $100 \mathrm{~mL}$ soil sample was mixed in 300 $\mathrm{mL}$ water in a $1000 \mathrm{~mL}$ measuring cup. Soil suspensions were mixed well by stirring. The floating particles and suspensions were filtered through $180-\mu \mathrm{m}$-pore and 450 $\mu \mathrm{m}$-pore sieves, 3 times. The residue on a $180-\mu \mathrm{m}$-pore sieve was rinsed with a fine water flow into a beaker, filtered with a screen cloth, and dried. Each soil sample was prepared 3 times.

\section{Preparation of egg suspension}

Under a stereomicroscope, full cysts were placed in a Petri dish and crushed, releasing eggs and juveniles (J2). The eggs and juveniles (J2) were transferred to a breaker and diluted to a final density of $1 \times 10^{6} \cdot \mathrm{L}^{-1}$.

\section{Inoculation}

Plastic cups (6.4-cm diameter, $17-\mathrm{cm}$ high) with bottom punched were filled with sterilized soil and sand (1:3). For germination, surface-sterilized seeds of each soybean line were placed on filter paper under dark conditions for $48 \mathrm{~h}$. One $2-3 \mathrm{~cm}$ long soybean seedling was then sown into each pre-irrigated plastic cup. After 3 days, a 2-mL egg suspension was injected into a $3-\mathrm{cm}$ hole around the root of the seedling. There were 5 replicate cups for each line. The cups were placed in large plastic boxes according to sample origin and these boxes were placed in a greenhouse at 27 $29^{\circ} \mathrm{C}$. Plants were watered daily.

\section{Microscopic examination}

After 30-35 days, the tops of the seedlings were removed. The soil and roots in the plastic cups were poured into a pot and then sprayed with a strong stream of water to dislodge the cysts. The cysts were isolated as described above and counted using a stereomicroscope.

\section{Identification}

Virulence differentiation of SCN population was based on the difference in reproductive ability on indicator lines. Based on the average number of cysts formed on the indicator lines, Female Indices (FI) was calculated, FI $\geq 10$ meant ' + ', FI $<10$ meant ' - '. Virulence phenotypes were identified by the race scheme and HG Type scheme. The formula of the female index (FI):

$$
\mathrm{FI}=\frac{\text { Number of cysts and femalesper plant }}{\text { Average number of cysts and femaleson Lee } 74} \times 100
$$

\section{Statistical analysis}

The correlation analysis among indicator lines was performed using IBM S.P.S.S. Statistics 21.

\section{Results}

\section{Distribution and Density of SCN in Jilin province}

Out of the 141 samples, 104 samples from all 38 cities (counties, town) were positive for SCN (73.76\%) (Fig. 1). 
Table 1: Densities of Heterodera glycines in Jilin province

\begin{tabular}{|c|c|c|c|c|c|}
\hline \multicolumn{2}{|c|}{ Sampling point city (County) } & \multirow{2}{*}{$\begin{array}{l}\text { Number of } \\
\text { samples }\end{array}$} & \multirow{2}{*}{$\begin{array}{l}\text { Number of samples } \\
\text { with SCN }\end{array}$} & \multirow{2}{*}{$\begin{array}{l}\text { Effective sample number } \\
(\geq 5 \text { cyst } / 100 \mathrm{~mL} \text { soil })\end{array}$} & \multirow{2}{*}{$\begin{array}{l}\text { Cyst density } \\
\text { (cysts/100 mL soil) }\end{array}$} \\
\hline City & County(town) & & & & \\
\hline \multirow[t]{4}{*}{ Baishan } & Baishan & 5 & 2 & 0 & 0.4 \\
\hline & Jingyu & 2 & 2 & 2 & 15 \\
\hline & Fusong & 4 & 3 & 0 & 1.75 \\
\hline & Changbai Korean Autonomous County & 4 & 3 & 1 & 2 \\
\hline \multirow[t]{2}{*}{ Tonghua } & Meihekou & 3 & 2 & 2 & 12.67 \\
\hline & Liuhe & 3 & 1 & 0 & 1 \\
\hline \multirow[t]{2}{*}{ Liaoyuan } & Dongliao & 3 & 1 & 1 & 1.67 \\
\hline & Dongfeng & 3 & 1 & 0 & 0.67 \\
\hline \multirow[t]{4}{*}{ Baicheng } & Baicheng & 3 & 3 & 3 & 14.67 \\
\hline & Taonan & 4 & 4 & 4 & 36.25 \\
\hline & Tongyu & 3 & 3 & 3 & 18.33 \\
\hline & Daan & 4 & 3 & 1 & 2.5 \\
\hline \multirow[t]{4}{*}{ Siping } & Gongzhuling & 5 & 5 & 4 & 19.8 \\
\hline & Shuangliao & 3 & 3 & 3 & 11.33 \\
\hline & Lishu & 4 & 3 & 2 & 3.75 \\
\hline & Yitong & 4 & 2 & 0 & 1.5 \\
\hline \multirow[t]{3}{*}{ Songyuan } & Fuyu & 4 & 3 & 1 & 2.5 \\
\hline & Changling & 4 & 3 & 1 & 3.25 \\
\hline & Qian’an & 4 & 3 & 1 & 2 \\
\hline \multirow[t]{5}{*}{ Jilin } & Jilin & 2 & 2 & 2 & 15.5 \\
\hline & Jiaohe & 2 & 2 & 2 & 11.5 \\
\hline & Huadian & 4 & 2 & 0 & 1.25 \\
\hline & Yongji & 4 & 4 & 2 & 4 \\
\hline & Panshi & 3 & 3 & 3 & 12.67 \\
\hline Yanbian $\quad$ Korean & Dunhua & 6 & 5 & 2 & 3.33 \\
\hline \multirow{7}{*}{ Autonomous Prefecture } & Wangqing & 4 & 3 & 0 & 1.75 \\
\hline & Antu & 4 & 3 & 2 & 3.75 \\
\hline & Helong & 4 & 3 & 1 & 2.25 \\
\hline & Yanji & 3 & 3 & 0 & 2 \\
\hline & Tumen & 4 & 4 & 2 & 3.5 \\
\hline & Hunchun & 5 & 3 & 0 & 1.2 \\
\hline & Longjing & 3 & 3 & 3 & 13.67 \\
\hline \multirow[t]{6}{*}{ Changchun } & Yushu & 4 & 2 & 1 & 2.25 \\
\hline & Changchun & 4 & 4 & 2 & 4.75 \\
\hline & Dehui & 4 & 3 & 0 & 1.75 \\
\hline & Nongan & 4 & 3 & 1 & 2.75 \\
\hline & Jiutai & 4 & 1 & 0 & 0.25 \\
\hline & Shuangyang & 4 & 1 & 1 & 1.25 \\
\hline Total/Average & & 141 & 104 & 53 & 6.33 \\
\hline
\end{tabular}

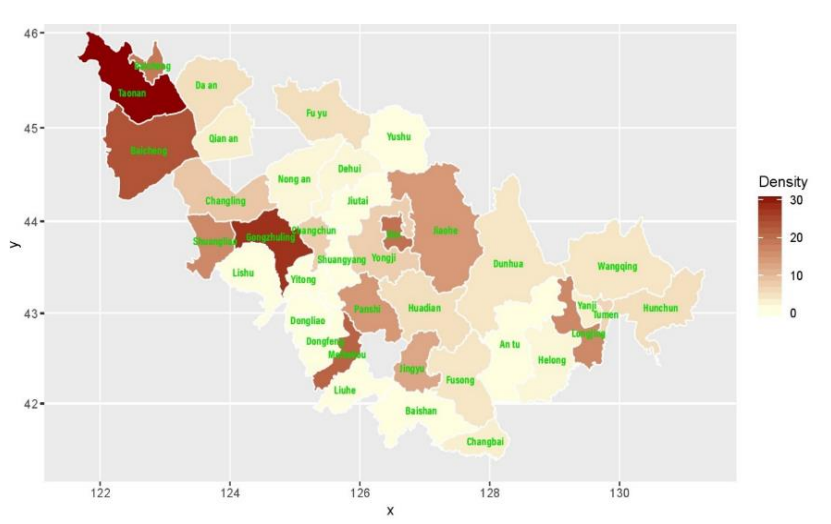

Fig. 1: Distribution of soybean cyst nematode (Heterodera glycines) in Jilin province

$100 \%$ of soil samples from Gongzhuling, Baishan, Yanji, Taonan, Tongyu and other 9 counties (cities) were positive for SCN.
The average cyst density of all soil samples was 6.33 cyst/100 mL. There were more than 10 cysts $/ 100 \mathrm{~mL}$ soil in 24 soil samples from 11 cities (counties, towns), especially in samples from the cities of Taonan, Baicheng, Gongzhuling and Jilin, as well as Tongyu county. The cyst density in Taonan county was 28 cysts $/ 100 \mathrm{~mL}$ soil. There were more than 5 cysts $/ 100 \mathrm{~mL}$ soils in 53 soil samples from 27 cities (counties, towns).

\section{Race identification}

Virulence phenotypes of 53 populations with more than 5 cysts $/ 100 \mathrm{~mL}$ soil were tested by race scheme. Seven races were identified, i.e., 1, 3, 5, 6, 9, 10 and 14 (Table 2). Race 3 was the most widely distributed, accounting for $62.26 \%$, followed by race 1 , accounting for $11.32 \%$. Race 5 and 6 accounted for $9.43 \%$ respectively. The SCN in one soil sample was race 10 , and one was race 14 ; of these, race 10 was first found in China. 
Yan et al. / Intl J Agric Biol, Vol 25, No 3, 2021

Table 2: Virulence phenotypes of Heterodera glycines in Jinlin province

\begin{tabular}{|c|c|c|c|c|c|c|c|c|c|c|c|c|c|c|c|c|c|c|c|c|}
\hline \multirow[b]{2}{*}{ West } & \multicolumn{2}{|c|}{ No. City } & \multirow{2}{*}{$\begin{array}{l}\text { County(town) } \\
\text { Fuyu }\end{array}$} & Pickett & \multicolumn{2}{|c|}{ Peking(1) } & \multicolumn{2}{|c|}{ PI88788(2) } & \multicolumn{2}{|c|}{ PI90763(3) } & \multicolumn{2}{|c|}{ PI437654(4) } & \multicolumn{2}{|c|}{ PI209332(5) } & \multicolumn{2}{|c|}{ PI89772(6) } & \multicolumn{2}{|c|}{ PI548316(7) } & \multirow{2}{*}{$\begin{array}{l}\text { Race } \\
3\end{array}$} & HG Type \\
\hline & 1 & Songyuan & & 3.31 & -2.71 & - & 1.51 & $-\quad($ & 0.60 & - & 5.72 & - & 7.83 & - & 0.90 & - & 6.63 & - & & 0 \\
\hline & 2 & & Changling & 3.55 & -1.66 & - & 7.58 & - & 4.98 & - & 1.90 & - & 7.82 & - & 2.61 & - & 19.67 & + & 3 & 7 \\
\hline & 3 & & Qian'an & 7.47 & -2.67 & - & 6.93 & - & 3.47 & - & 0.80 & - & 7.73 & - & 4.00 & - & 25.87 & + & 3 & 7 \\
\hline & 4 & Baicheng & Taonan & 41.80 & +3.28 & - & 2.62 & - & 25.25 & + & 13.93 & + & 11.48 & + & 3.93 & - & 29.51 & + & 10 & $3,4,5,7$ \\
\hline & 5 & & Taonan & 18.22 & +4.12 & - & 2.82 & - & 2.17 & - & 0.43 & - & 16.70 & + & 4.34 & - & 37.96 & + & 6 & 5.7 \\
\hline & 6 & & Taonan & 1.33 & -2.92 & - & 2.92 & - & 4.77 & - & 4.51 & - & 0.80 & - & 2.12 & - & 7.16 & - & 3 & 0 \\
\hline & 7 & & Taonan & 8.41 & -1.68 & - & 6.25 & - & 5.29 & - & 2.16 & - & 2.16 & - & 4.33 & - & 8.65 & - & 3 & 7 \\
\hline & 8 & & Tongyu & 3.77 & -2.08 & - & 0.19 & - & 2.26 & - & 5.66 & - & 4.91 & - & 5.09 & - & 23.58 & + & 3 & 7 \\
\hline & 9 & & Tongyu & 0.98 & -3.58 & - & 5.21 & - & 4.56 & - & 4.56 & - & 2.28 & - & 5.86 & - & 6.51 & - & 3 & 0 \\
\hline & 10 & & Tongyu & 8.20 & -3.61 & - & 7.87 & - & 4.92 & - & 1.97 & - & 5.25 & - & 2.62 & - & 6.56 & - & 3 & 0 \\
\hline & 11 & & Baicheng & 4.61 & -1.05 & - & 1.05 & - & 1.26 & - & 1.05 & - & 3.14 & - & 0.84 & - & 1.68 & - & 3 & 5.7 \\
\hline & 12 & & Baicheng & 11.78 & +11.36 & + & 3.93 & - & 7.02 & - & 1.45 & - & 15.50 & + & 2.48 & - & 30.37 & + & 9 & 1.5 .7 \\
\hline & 13 & & Baicheng & 4.86 & -2.13 & - & 3.04 & - & 2.74 & - & 3.95 & - & 9.12 & - & 2.43 & - & 27.66 & + & 3 & 7 \\
\hline & 14 & & Daan & 14.60 & +2.54 & - & 12.38 & + & 1.59 & - & 0.63 & - & 13.33 & + & 4.44 & - & 20.95 & + & 5 & 2.5 .7 \\
\hline East & 15 & Yanbian & Longjing & 13.24 & +11.35 & + & 1.89 & - & 2.16 & - & 1.08 & - & 1.62 & - & 0.00 & - & 13.24 & + & 9 & 1,7 \\
\hline & 16 & Korean & Longjing & 3.27 & -0.30 & - & 5.36 & - & 2.38 & - & 4.76 & - & 3.27 & - & 2.68 & - & 23.21 & + & 3 & 7 \\
\hline & 17 & Autonomous & Longjing & 2.32 & - 0.70 & - & 3.25 & - & 2.55 & - & 2.32 & - & 2.32 & - & 2.55 & - & 3.02 & - & 3 & 0 \\
\hline & 18 & Prefecture & Duhua & 8.82 & -1.26 & - & 1.76 & - & 8.82 & - & 4.03 & - & 2.02 & - & 8.06 & - & 8.56 & - & 3 & 0 \\
\hline & 19 & & Duhua & 7.21 & -4.08 & - & 0.31 & - & 0.94 & - & 0.00 & - & 3.45 & - & 7.21 & - & 15.05 & + & 3 & 7 \\
\hline & 20 & & Helong & 12.70 & +2.78 & - & 2.78 & - & 7.14 & - & 7.54 & - & 7.94 & - & 2.38 & - & 9.13 & - & 6 & 0 \\
\hline & 21 & & Antu & 11.18 & +13.21 & + & 4.47 & - & 12.60 & + & 3.25 & - & 7.72 & - & 6.71 & - & 34.96 & + & 14 & 1.3 .7 \\
\hline & 22 & & Antu & 7.59 & -1.31 & - & 11.26 & + & 2.36 & - & 4.97 & - & 2.36 & - & 0.79 & - & 32.46 & + & 1 & 2.7 \\
\hline & 23 & & Tumen & 12.31 & +2.24 & - & 19.78 & + & 5.60 & - & 2.61 & - & 28.36 & + & 4.10 & - & 16.42 & + & 5 & 2.5 .7 \\
\hline & 24 & & Tumen & 0.56 & -2.52 & - & 3.36 & - & 2.80 & - & 4.48 & - & 5.04 & - & 4.48 & - & 15.41 & + & 3 & 7 \\
\hline Mid- & 25 & Jilin & Jilin & 36.04 & +1.90 & - & 2.17 & - & 2.44 & - & 1.36 & - & 34.42 & + & 2.17 & - & 27.37 & + & 6 & 5.7 \\
\hline & 26 & & Jilin & 15.58 & +3.90 & - & 13.96 & + & 1.62 & - & 11.36 & + & 6.17 & - & 1.30 & - & 20.45 & + & 5 & 2.4 .7 \\
\hline & 27 & & Jiaohe & 3.27 & -1.76 & - & 1.01 & - & 2.26 & - & 0.75 & - & 1.01 & - & 2.76 & - & 31.91 & + & 3 & 7 \\
\hline & 28 & & Jiaohe & 4.82 & -1.29 & - & 11.58 & + & 2.25 & - & 6.11 & - & 3.54 & - & 9.32 & - & 15.43 & + & 1 & 2.7 \\
\hline & 29 & & Yongji & 7.51 & -3.18 & - & 2.31 & - & 4.34 & - & 2.60 & - & 8.96 & - & 2.02 & - & 6.07 & - & 3 & 0 \\
\hline & 30 & & Yongji & 5.65 & -4.32 & - & 5.98 & - & 4.32 & - & 0.00 & - & 3.65 & - & 7.64 & - & 8.31 & - & 3 & 0 \\
\hline & 31 & & Panshi & 1.61 & -0.64 & - & 16.40 & + & 1.29 & - & 0.64 & - & 4.82 & - & 2.89 & - & 19.61 & + & 1 & 2 \\
\hline & 32 & & Panshi & 6.21 & - 3.92 & - & 0.33 & - & 3.59 & - & 3.27 & - & 1.96 & - & 3.92 & - & 26.80 & + & 3 & 7 \\
\hline & 33 & & Panshi & 3.79 & -2.84 & - & 4.73 & - & 4.42 & - & 3.15 & - & 5.99 & - & 4.73 & - & 38.49 & + & 3 & 7 \\
\hline & 34 & Siping & Gongzhuling & 7.24 & - 4.91 & - & 7.49 & - & 7.75 & - & 7.49 & - & 5.94 & - & 1.55 & - & 14.21 & + & 3 & 7 \\
\hline & 35 & & Gongzhuling & 7.07 & - 10.61 & + & 5.14 & - & 5.47 & - & 7.07 & - & 9.32 & - & 7.72 & - & 28.94 & + & 3 & 7 \\
\hline & 36 & & Gongzhuling & 3.97 & - 3.44 & - & 4.76 & - & 3.44 & - & 4.23 & - & 6.88 & - & 7.94 & - & 9.79 & - & 3 & 0 \\
\hline & 37 & & Gongzhuling & 7.31 & -6.15 & - & 15.77 & + & 3.46 & - & 5.77 & - & 6.92 & - & 4.23 & - & 25.00 & + & 1 & 2.7 \\
\hline & 38 & & Lishu & 6.68 & -1.73 & - & 3.22 & - & 3.96 & - & 2.48 & - & 14.11 & + & 3.22 & - & 21.04 & + & 3 & 5.7 \\
\hline & 39 & & Lishu & 2.25 & -2.46 & - & 2.66 & - & 3.89 & - & 2.25 & - & 0.41 & - & 3.69 & - & 30.12 & + & 3 & 7 \\
\hline & 40 & & Shuangliao & 0.00 & -0.00 & - & 3.59 & - & 5.25 & - & 1.66 & - & 2.49 & - & 5.25 & - & 22.38 & + & 3 & 7 \\
\hline & 41 & & Shuangliao & 0.27 & - 0.00 & - & 2.73 & - & 6.01 & - & 1.37 & - & 3.28 & - & 2.46 & - & 3.55 & - & 3 & 0 \\
\hline & 42 & & Shuangliao & 2.30 & - 0.29 & - & 4.02 & - & 2.30 & - & 3.45 & - & 2.01 & - & 2.01 & - & 4.02 & - & 3 & 0 \\
\hline & 43 & Changchun & Changchun & 20.90 & +6.35 & - & 11.64 & + & 2.65 & - & 2.38 & - & 17.72 & + & 6.35 & - & 4.50 & - & 5 & 2.5 \\
\hline & 44 & & Changchun & 3.61 & -4.26 & - & 6.56 & - & 4.92 & - & 7.21 & - & 9.18 & - & 4.92 & - & 33.44 & + & 3 & 7 \\
\hline & 45 & & Shuangyang & 6.39 & -1.53 & - & 6.91 & - & 6.91 & - & 1.02 & - & 3.32 & - & 5.12 & - & 19.44 & + & 3 & 7 \\
\hline & 46 & & Yushu & 2.97 & - 2.97 & - & 6.53 & - & 4.75 & - & 1.19 & - & 7.72 & - & 5.93 & - & 6.82 & - & 3 & 0 \\
\hline & 47 & & Nong`an & 12.15 & +0.00 & - & 3.43 & - & 4.36 & - & 0.62 & - & 4.36 & - & 4.36 & - & 7.79 & - & 6 & 0 \\
\hline & 48 & Baishan & Jingyu & 8.90 & -4.71 & - & 2.62 & - & 4.71 & - & 7.59 & - & 4.45 & - & 6.28 & & 8.12 & - & 3 & 0 \\
\hline & 49 & & Jingyu & 0.85 & -2.56 & - & 14.25 & + & 0.57 & - & 0.57 & - & 15.67 & + & 3.70 & - & 27.92 & + & 1 & 2.5 .7 \\
\hline & 50 & & $\begin{array}{l}\text { Changbai Korean } \\
\text { Autonomous }\end{array}$ & 3.77 & - 3.77 & - & 3.19 & - & 0.00 & - & 5.80 & - & 1.45 & - & 1.74 & - & 7.25 & - & 3 & 0 \\
\hline & 51 & Tonghua & Meihekou & 13.33 & +4.67 & - & 4.00 & - & 5.33 & - & 4.00 & - & 5.67 & - & 0.33 & - & 22.00 & + & 6 & 7 \\
\hline & 52 & & Meihekou & 17.59 & +6.19 & - & 16.29 & + & 0.98 & - & 4.89 & - & 3.26 & - & 0.33 & - & 33.55 & + & 5 & 2.5 .7 \\
\hline & 53 & Liaoyuan & Dongliao & 2.67 & - 0.00 & - & 13.07 & + & 2.67 & - & 0.27 & - & 12.27 & + & 4.27 & & 6.40 & - & 1 & 2.5 \\
\hline
\end{tabular}

Note: '+': FI $\geq 10$; '-': FI $<10$.

\section{HG Type}

SCN virulence phenotypes in 53 soil samples with more than 5 cysts $/ 100 \mathrm{~mL}$ soil were tested using HG Type scheme. We identified 12 HG Types 0, 2, 2.5, 2.7, 5.7, 7, 1.7, 1.3.7, 1.5.7, 2.4.7, 2.5.7, 3.4.5.7, (Table 2). HG Type 7 and 0 was predominant, accounting for $33.96 \%$ and $30.18 \%$. Both HG Type 5.7 and HG Type 2.5.7 accounted for 7.55\%. HG Type 2.5 and HG Type 2.7 accounted for $3.77 \%$ and $5.66 \%$. The remaining types each accounted for $1.89 \%$. HG Type 1.7 was first time recorded in China and HG Type 3.4.5.7 was the first found worldwide.

\section{Virulence of SCN groups on indicator lines}

33 SCN populations in this study were virulent on PI548316: $62.26 \%$ had a FI $\geq 10$ on the indicator line (\#7), $26.42 \%$ FI $\geq 10$ on Pickett, $20.75 \%$ FI $\geq 10$ on PI88788 (\#2) and $18.87 \%$ FI $\geq 10$ on PI209332 (\#5). PI89772 had the most resistance: $100 \%$ populations had a FI $<10$ on the line (\#6). 3.78\% had a FI $\geq 10$ on PI 90763 (\#3), PI 437654 (\#4) and $7.55 \%$ on Peking (\#1), (Table 2).

A highly significant positive correlation $(P<0.01)$ was found between the FI on PI 90763 (\#3) and PI 437654 (\#4), as were correlations among Pickett, PI 90763 (\#3) and 
Table 3: Correlation coefficients among soybean differential lines with resistance to Heterodera glycines based on female indices (FIs) from 53 populations in Jilin province

\begin{tabular}{|c|c|c|c|c|c|c|c|c|}
\hline Correlation coefficients & Pickett & Peking & PI88788 & PI90763 & PI437654 & PI209332 & PI89772 & PI548316 \\
\hline Pickett & 1 & .254 & .038 & $.479^{* *}$ & $.301^{*}$ & $.555^{* *}$ & -.099 & .253 \\
\hline Peking & & 1 & -.014 & .202 & .147 & .106 & .074 & $.295^{*}$ \\
\hline PI88788 & & & 1 & -.168 & .013 & $.282^{*}$ & .002 & .114 \\
\hline PI437654 & & & & & 1 & -.098 & -.041 & .110 \\
\hline PI209332 & & & & & & 1 & .015 & .224 \\
\hline PI89772 & & & & & & & 1 & -.024 \\
\hline PI548316 & & & & & & & & 1 \\
\hline
\end{tabular}

$* P<0.05, * * P<0.01$

Table 4: Correspondence between race and HG Type

\begin{tabular}{|c|c|c|c|c|c|c|c|c|c|c|c|c|c|c|c|}
\hline Race & & & & & & & & & HG Type & & & & & & \\
\hline 1 & 2 & 2.4 & 2.5 & 2.6 & 2.7 & 2.4 .5 & 2.4 .6 & 2.4 .7 & 2.5 .6 & 2.5 .7 & 2.6 .7 & 2.4 .5 .6 & 2.4 .5 .7 & 2.5 .6 .7 & 2.4 .5 .6 .7 \\
\hline 2 & 1.2 & 1.2 .4 & 1.2 .5 & 1.2 .6 & 1.2 .7 & 1.2 .4 .5 & 1.2 .4 .6 & 1.2.4.7 & 1.2 .5 .6 & 1.2.5.7 & 1.2 .6 .7 & 1.2.4.5.6 & 1.2.4.5.7 & 1.2 .5 .6 .7 & 1.2.4.5.6.7 \\
\hline 3 & 0 & 4 & 5 & 6 & 7 & 4.5 & 4.6 & 4.7 & 5.6 & 5.7 & 6.7 & 4.5 .6 & 4.5 .7 & 5.6 .7 & 4.5.6.7 \\
\hline 4 & 1.2 .3 & 1.2.3.4 & 1.2.3.5 & 1.2.3.6 & 1.2.3.7 & 1.2 .3 .4 .5 & 1.2.3.4.6 & 1.2.3.4.7 & 1.2.3.5.6 & 1.2 .3 .5 .7 & 1.2.3.6.7 & 1.2.3.4.5.6 & 1.2.3.4.5.7 & 1.2 .3 .5 .6 .7 & 1.2 .3 .4 .5 .6 .7 \\
\hline 5 & 2 & 2.4 & 2.5 & 2.6 & 2.7 & 2.4 .5 & 2.4 .6 & 2.4 .7 & 2.5 .6 & 2.5 .7 & 2.6 .7 & 2.4 .5 .6 & 2.4.5.7 & 2.5 .6 .7 & 2.4.5.6.7 \\
\hline 6 & 0 & 4 & 5 & 6 & 7 & 4.5 & 4.6 & 4.7 & 5.6 & 5.7 & 6.7 & 4.5 .6 & 4.5 .7 & 5.6 .7 & 4.5 .6 .7 \\
\hline 7 & 2.3 & 2.3 .4 & 2.3 .5 & 2.3 .6 & 2.3 .7 & 2.3.4.5 & 2.3 .4 .6 & 2.3.4.7 & 2.3 .5 .6 & 2.3.5.7 & 2.3 .6 .7 & 2.3.4.5.6 & 2.3.4.5.7 & 2.3.5.6.7 & 2.3.4.5.6.7 \\
\hline 8 & 3 & 3.4 & 3.5 & 3.6 & 3.7 & 3.4 .5 & 3.4 .6 & 3.4 .7 & 3.5 .6 & 3.5 .7 & 3.6 .7 & 3.4 .5 .6 & 3.4 .5 .7 & 3.5.6.7 & 3.4.5.6.7 \\
\hline 9 & 1 & 1.4 & 1.5 & 1.6 & 1.7 & 1.4 .5 & 1.4 .6 & 1.4 .7 & 1.5 .6 & 1.5 .7 & 1.6 .7 & 1.4 .5 .6 & 1.4.5.7 & 1.5 .6 .7 & 1.4 .5 .6 .7 \\
\hline 10 & 3 & 3.4 & 3.5 & 3.6 & 3.7 & 3.4 .5 & 3.4 .6 & 3.4 .7 & 3.5 .6 & 3.5 .7 & 3.6 .7 & 3.4 .5 .6 & 3.4 .5 .7 & 3.5.6.7 & 3.4.5.6.7 \\
\hline 11 & 1.2 & 1.2 .4 & 1.2 .5 & 1.2 .6 & 1.2 .7 & 1.2.4.5 & 1.2 .4 .6 & 1.2.4.7 & 1.2 .5 .6 & 1.2.5.7 & 1.2.6.7 & 1.2 .4 .5 .6 & 1.2.4.5.7 & 1.2 .5 .6 .7 & 1.2.4.5.6.7 \\
\hline 12 & 1.3 & 1.3.4 & 1.3 .5 & 1.3 .6 & 1.3 .7 & 1.3.4.5 & 1.3.4.6 & 1.3.4.7 & 1.3.5.6 & 1.3.5.7 & 1.3.6.8 & 1.3.4.5.6 & 1.3.4.5.7 & 1.3.5.6.7 & 1.3.4.5.6.7 \\
\hline 13 & 1 & 1.4 & 1.5 & 1.6 & 1.7 & 1.4 .5 & 1.4 .6 & 1.4 .7 & 1.5 .6 & 1.5 .7 & 1.6 .7 & 1.4 .5 .6 & 1.4 .5 .7 & 1.5 .6 .7 & 1.4.5.6.7 \\
\hline 14 & 1.3 & 1.3 .4 & 1.3 .5 & 1.3 .6 & 1.3 .7 & 1.3.4.5 & 1.3.4.6 & 1.3.4.7 & 1.3.5.6 & 1.3.5.7 & 1.3.6.8 & 1.3.4.5.6 & 1.3.4.5.7 & 1.3.5.6.7 & 1.3.4.5.6.7 \\
\hline 15 & 2.3 & 2.3 .4 & 2.3 .5 & 2.3 .6 & 2.3 .7 & 2.3 .4 .5 & 2.3 .4 .6 & 2.3 .4 .7 & 2.3 .5 .6 & 2.3 .5 .7 & 2.3.6.7 & 2.3.4.5.6 & 2.3.4.5.7 & 2.3.5.6.7 & 2.3.4.5.6.7 \\
\hline 16 & 1.2 .3 & 1.2.3.4 & 1.2.3.5 & 1.2.3.6 & 1.2.3.7 & 1.2.3.4.5 & 1.2.3.4.6 & 1.2.3.4.7 & 1.2.3.5.6 & 1.2.3.5.7 & 1.2.3.6.7 & 1.2.3.4.5.6 & 1.2.3.4.5.7 & 1.2.3.5.6.7 & 1.2.3.4.5.6.7 \\
\hline
\end{tabular}

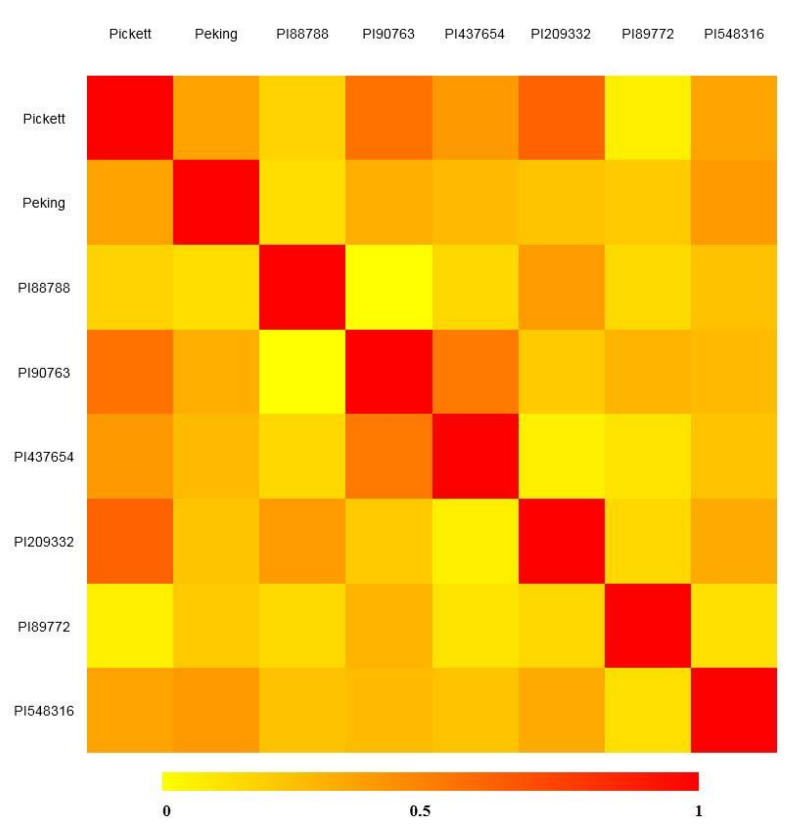

Fig. 2: Heat map for correlation between indicator lines

PI 209332 (\#5). Pickett and PI 437654 (\#4) were positively correlated $(P<0.05)$. PI $88788(\# 2)$ and PI209332 (\#5) were also positively correlated (Table 3 ). Additional correlation was found between Peking (\#1) and PI548316 (\#7) (Fig. 2).

\section{Discussion}

In a previous survey conducted in $1988, \mathrm{SCN}$ was distributed over $83 \%$ of Jilin province, especially in the counties of Zhenlai, Yushu, Tongyu and Jingyu and in Baicheng city (Liu and Wu 1988). A total of 38 (100\%) cities (counties, towns) were infested with SCN in this study. Taonan, Baicheng, Gongzhuling, Jilin city and Tongyu county are the hotspots for SCN distribution (Fig. 1), the same as previous survey, except Jilin city. Significantly, SCN was not detected in Jilin and Longjing cities in the survey conducted in 1988 and cyst density of sample from Gongzhuling increased, possibly due to the long-term cultivation of soybeans and the lack of disease resistant varieties in these regions. As shown in Fig. 1, the density of SCN was the highest and widely distributed in the western of Jilin Province, which may be due to the most of saline-alkali land, followed by the middle part, the lowest in the east.

According to similarities of cellular resistance response in tests, soybean resistance to $\mathrm{SCN}$ was classified into two main types: the PI 88788-type, including PI 209332 and PI 548316; and the Peking-type, including PI 90763, PI 89772 and perhaps, PI 437654 (Endo 1965; Kim et al. 1987; Halbrendt et al. 1992; Mahalingham and Skorupska 1996). The results of this study are similar to and different from those of previous studies. The resistance of PI 209332 was similar to that of PI 88788 and PI 548316 (Anand 1992). $H$. glycines reproduction on these three differentials was 
significantly positively correlated (Zheng et al. 2006; Colgrove and Niblack 2008; Acharya et al. 2016). In this study, PI 209332 (\#5) and PI88788 (\#2) had positive correlation on FI $(P<0.05)$, but not PI 548316 (\#7) (Fig. 2). What's interesting was that significant correction was found between PI 548316 and Peking in this study. PI 548316, originally assigned to PI88788-type, now was classified into the Peking-type. Differentials PI 90763 (\#3) and PI 437654 (\#4) exhibited significant positive correlations on FI $(P<$ 0.01), but not Peking. PI 90763 and PI 437654 were not classified into the Peking-type now. An observation of a stage-related effect of resistance on juvenile development supports that the resistance response may be different between the types (Halbrendt et al. 1992). In addition, Pickett is considered to be the progeny of Peking and may carry similar resistance genes. However, in this study, Pickett and PI 90763 (\#3), PI 437654 (\#4), PI 209332 (\#5) were found to have significant positive correlation but not Peking. This result may be due to the high genetic diversity of the field population. Pickett should be used to distinguish the virulence phenotypes of SCN field populations in Jilin province.

Virulence phenotypes of $H$. glycines populations were evaluated by race scheme and HG Type scheme. A total of 7 races and $12 \mathrm{HG}$ Types were identified, and race 10 and HG Type 1.7 were first recorded in China. In 1988, race 10 was first discovered in Arkansas (Riggs and Schmitt 1988). In 1996, the race was also identified in Ohio (Willson et al. 1996). HG Type 3.4.5.7 was first found worldwide.

If Jilin Province is divided into eastern, western and central regions, race 1, 3, 5, 6 are distributed in all the three regions in common. Race 1 is dominantly distributed in the mid-region, while the other three races are common. Race 9 was found sporadically in the east and west, but not in the central Jilin Province. Race 10 and 14 distributed in one sampling point in the West and East respectively.

A population`s HG Type name can clearly show invalid indicator plants. Any cultivar that has a similar FI $\geq 10$ should not be used in a field that has an $H$. glycines population that is compatible with that indicator line. In this student (Table 2), due to FI $\geq 10$ on PI 548316 and Pickett in most of populations, the use of PI 548316 and Pickett as resistant parents should be minimized in Jilin province. The use of PI 88788 (\#2) should be also reduced in the west. PI 89772, PI 437654 and PI 90763 are better sources for resistance to SCN. Recently, multi resistant varieties, such as Kangxian 3 (Cao et al. 2014), Dongnong L-10 (Wu et al. 2016), ZDD24656, a variety derived from PI 437654 and ZDD2315 (Lian et al. 2017), Andou 162, a descendant of Franklin, Bedford and Hartwig (Wang et al. 2019), Zhonghuang 26 and Zhonghuang 54, varieties derived from PI 437654 (Wang and Wang 2016) can be used as source of resistance.

When comparing the two schemes, the same race corresponded to multiple HG types, e.g., race 3 corresponded to HG Type 7 and HG Type 5.7 in this study.
Additionally, the same HG type may also correspond to multiple races, e.g., HG Type 7 corresponded to race 3 and race 6, and HG Type 5.7 also corresponded to race 3 and race 6 in this study. In a previous study (Wang 2015), HG Type 2.7 corresponded to race 1 and race 5 . We considered the SCN population with HG Type 2.7 (race 1) in this study was different from the population with HG Type 2.7 (race 5) in the previous study (Wang 2015). Possibly, that was the same as the population with HG Type 2.7 (race 1) in the previous study. In this way, neither scheme could completely distinguish the virulence phenotypes of SCN populations. HG Type testing is internationally accepted because it is relatively accurate and easily expandable as new soybean germplasm is released and deployed (Winter $e t$ al. 2006). The resistant line PI 438489B (Yue et al. 2001) was added to the set of differentials used in the HG Type test in 2007 (Mitchum et al. 2007). However, as the number of identified hosts increases, HG type testing will become more complex. Currently, HG Type + Race are used in SCN research fields (Han et al. 2015; Lin et al. 2016).

Although there is no one-to-one correspondence between race and HG type, there are some corresponding relationships (Table 4). Theoretically, 128 HG types are possible with HG Type testing. Each race corresponded to $16 \mathrm{HG}$ types, and each two races corresponded to the same HG type, e.g., race 3 and race 6 corresponded to the same 16 HG Types. Accordingly, in theory, 256 different SCN virulence types are possible with the combination of the two methods, but the lack of characterization of them. More trials are needed for further validation. According to this study, the combination of the two methods was suitable for the study of genetic diversity of SCN in Jilin province. Pickett which was removed in HG scheme should be included not as an indicator line but just to separate different races from the same HG type in Jilin province.

\section{Conclusion}

In this study, 104 soil samples from 38 cites (cities, counties and towns) were positive for SCN across Jilin province 7 races and $12 \mathrm{HG}$ types were identified, with Race 3 and HG Type 7 being the most dominant genotypes. PI 90763, PI 437654 and PI89772 were recommended as excellent sources against cyst nematode. It suggests that Peking-type resistance sources were preferred to pi88788-type in Jilin province. The combination of the race and $\mathrm{HG}$ scheme is recommended for studying the genetic diversity of SCN in Jilin province. That is, Pickett should be included not as an indicator line but just to separate different races from the same HG type.

\section{Acknowledgement}

We acknowledge the financial supports of the National Key Research and Development Program of China (Grant No. 2018YFD1000905). 


\section{Author Contributions}

Xiujuan Yan and Yuxi Duan conceived and designed the experiments; Qiang Qiu and Xiaofeng Zhu collected soil samples; Xiujuan Yan, Jinwen Liu and Mingshu Li performed the experiments; Jinwen Liu analyzed the data;Xiujuan Yan and Jinwen Liu wrote the paper.

\section{References}

Acharya K, C Tande, E Byamukama (2016). Determination of Heterodera glycines virulence phenotypes occurring in South Dakota. Plant Dis 100:2281-2286

Anand SC (1992). Registration of 'Delsoy 4710' soybean. Crop Sci 32:1294

Cao GL, X Zhao, Q Wang, XX Meng, SH Wei, YP Han, XX Wu, WB Li (2014). Resistance evaluation of soybean germplasm to races 1, 3 and 4 of soybean cyst nematode (Heterodera glycines). Soybean Sci 33:563-565

Chen JS, XB Li, ZY Li, CJ Zhou, X Luo, D Wang, YX Duan, LJ Chen (2015). Identification of the Virulence Type of Soybean Cyst Nematode under Continuous Cropping in Daqing and Anda. Soybean Sci 34:675-678

Colgrove AL, TL Niblack (2008). Correlation of female indices from virulence assays on inbred lines and field populations of Heterodera glycines. J Nematol 40:39-45

Cui H, CJ Li, YF Hu, YZ Mao, J You, MZ Wang, JS Chen, ZY Tian, CL Wang (2018). Identification of HG types of soybean cyst nematode Heterodera glycines and resistance screening on soybean genotypes in Northeast China. J Nematol 50:41-50

Endo BY (1965). Histological responses of resistant and susceptible soybean varieties, and backcross progeny to entry and development of Heterodera glycines. Phytopathology 55:375-381

Golden AM, JM Epps, RD Riggs, LA Duclos, JA Fox, RL Bernard (1970). Terminology and identity of infra specific forms of the soybean cyst nematode Heterodera glycines. Plant Dis Rep 54:544-546

Halbrendt J, S Lewis, E Shipe (1992). A technique for evaluating Heterodera glycines development in susceptible and resistant soybean. J Nematol 24:84-91

Han YP, X Zhao, GL Cao, Y Wang, YH Li, DY Liu, WL Teng, ZW Zhang, DM Li, LJ Qiu, HK Zheng, WB Li (2015). Genetic characteristics of soybean resistance to HG type 0 and $\mathrm{HG}$ type 1.2.3.5.7 of the cyst nematode analyzed by genome-wide association mapping. BMC Genomics 16; Article 598

Howland A, N Monnig, J Mathesius, M Nathan, MG Mitchum (2018). Survey of Heterodera glycines population densities and virulence phenotypes during 2015-2016 in Missouri. Plant Dis 102:2407-2410

Kim DG, IS Choi, WY Han, YH Ryu, MS Kim, CW Bae (2013). Studies on HG type of Heterodera glycines in Korea. Res Plant Dis 19:31-35

Kim DG, RD Riggs,RT Robbin, L Rakes (1997). Distribution of races of in the Central United States. J Nematol 29:173-179

Kim YH, RD Riggs, KS Kim (1987). Structural changes associated with resistance of soybean to Heterodera glycines. J Nematol 19:177-187

Lian Y, JQ Guo, HC Li, YK Wu, H Wei, JS Wang, JY Li, WG Lu (2017). A New Race (X12) of Soybean Cyst Nematode in China. J Nematol 49:321-326
Lin JY, M Mazarei, N Zhao, CN Hatcher, WA Wuddineh, M Rudis, TH Tschaplinski, VR Pantalone, PR Arelli, T Hewezi, F Chen, CN Stewart (2016). Transgenic soybean overexpressing GmSAMTI exhibits resistance to multiple-HG types of soybean cyst nematode Heterodera glycines. Plant Biotechnol J 14:2100-2109

Liu WZ (1995). Research Techniques of Plant Nematology, $1^{\text {st }}$ edn. Liaoming Science and Technology Press, Shenyang, China

Liu XM, X Wu (1988). Preliminary study on the distribution of soybean cyst nematode in Jilin Province. J Jilin Agric Univ 10:16-19

Mahalingham R, HT Skorupska (1996). Cytological expression of early response to infection by Heterodera glycines Ichinohe in resistant PI 437654 soybean. Genome 39:986-998

Mitchum MG, JA Wrather, RD Heinz, JG Shannon, G Danekas (2007). Variability in distribution and virulence phenotypes of Heterodera glycines in Missouri during 2005. Plant Dis 91:1473-1476

Niblack TL, J Wrather, R Heinz, P Donald (2003). Distribution and virulence phenotypes of Heterodera glycines in Missouri. Plant Dis 87:929-932

Niblack TL, PR Arelli, GR Noel, CH Ppperman, JH Orf, DP Schmitt, JG Shannon, GL Tylka (2002). A revised classification scheme for genetically diverse populations of Heterodera glycines. J Nematol 34:279-288

Riggs RD, DP Schmitt (1988). Complete characterization of the race scheme for Heterodera glycines. J Nematol 20:392-395

Rzodkiewicz PA (2010). Characterization of soybean cyst nematode diversity in Kansas. M.Sc. Thesis. Kansas State University, Manhattan, Kansas

Song MJ, XF Zhu, D Wang, WW Wang, LJ Chen, XY Liu, YX Duan (2016). Population distribution and pathogenicity differentiation of soybean cyst nematode in main soybean production areas of China. Soybean Sci 37:630-636

Wang D (2015). Population diversity and genetic differentiation of Heterodera glycines. M.Sc. Thesis, Shenyang Agricultural University, Shenyang, China

Wang D, XF Zhu, YY Wang, X Luo, P Song, F Zhu, F Wang, JS Chen, LJ Chen, YX Duan (2014). A reassessment of virulence phenotypes of soybean cyst nematode (Heterodera glycines) in China with Hgtyping method. Plant Dis 98:702

Wang JJ, JR Li, BX Zhang, RP Zang, XL Liu, RZ Zhang, DJ Wei, BS Yu (2019). Breeding of soybean variety Andou 162 with resistance to cyst nematode. Soybean Sci 38:836-838

Wang L, LZ Wang (2016). Breeding of resistance to soybean cyst nematode and breeding of cultivars- Zhonghuang 26 and Zhonghuang 54 Soybean Sci Technol 2:10-14

Willson HR, RM Riedel, JB Eisley, CE Young, JR Jasinski, TA Wheeler, PH Kauffman, PE Pierson, MC Stuart (1996). Distribution of Heterodera glycines in Ohio. J Nematol 28:599-603

Winter SM, JI Rajcan, BJ Shelp (2006). Soybean cyst nematode: Challenges and opportunities Can J Plant Sci 86:25-32

Wu DP, Y Zhao, BH Cheng, B Liu, YP Han, X Zhao, WB Li (2016). Genetic model analysis on resistance of cv. Dongnong L-10 to Race 3 of soybean cyst nematode. Soybean Sci 35:367-372

Xu YL, LF Wang, LL Zhan (2010). The research advances on soybean cyst nematodes. Soybean Technol 1:21-24

Yue P, PR Arelli, DA Sleper (2001). Molecular characterization of resistance to Heterodera glycines in soybean PI 438489B. Theor Appl Genet 102:921-928

Zheng JW, YH Li, SY Chen (2006). Characterization of the virulence phenotypes of Heterodera glycines in Minnesota. J Nematol 38:383-390 